\title{
Novos conceitos em modelamento corneano
}

\author{
New concepts in corneal reshaping
}

Daniela Coneglian Brocchetto ${ }^{1}$,Brunno Dantas², Paulo Schor $^{3}$

\section{ResUMo}

A córnea possui grande influência no processo visual. Porém é passível de deformação, tal como o aplanamento. Quando esse aplanamento é causado pelo uso de lentes de contato, chamamos de "corneal warpage". Neste caso, alguns sintomas podem ocorrer como visão embaçada com os óculos, hiperemia conjuntival, sensação de corpo estranho, etc. Pensava-se que a córnea era mais rígida e, com o tempo, percebeu-se que a córnea possui entidade viscoelástica. Podemos dizer que a córnea possui uma entidade biomecânica e que, quando sofre instabilidade, ocorrem algumas alterações, como no caso do ceratocone, onde ocorre uma degeneração progressiva corneana. Com o intuito de buscar a estabilização corneana, foi desenvolvida a técnica de "crosslink", que consiste em promover ligações covalentes entre as lamelas da córnea para torná-la mais firme e menos elástica. Outra alteração corneana pode ser induzida, de forma planejada, através do modelamento corneano com o uso de lentes de contato rígidas, com coeficiente de transmissibilidade de oxigênio (DK/L) maior. Elas são usadas somente durante o sono, não sendo necessário o seu uso diurno. Esta técnica é conhecida como Ortoceratologia e seus conceitos serão abordados neste estudo.

Descritores: Procedimentos ortoceratológicos; Ortoceratologia; Colágeno; Topografia da córnea; Córnea/fisiopatologia; Córnea/lesões; Ceratocone; Lentes de contato; Riboflavina/uso terapêutico; Terapia ultravioleta

\footnotetext{
${ }^{1}$ Tecnóloga em Oftalmologia, Pós-graduanda (Mestrado) em Lentes de Contato pela Universidade Federal de São Paulo - UNIFESP São Paulo (SP), Brasil;

${ }^{2}$ Pós-graduado em Oftalmologia pela Universidade Gama Filho - Rio de Janeiro (RJ), Brasil; Responsável pelo Setor de Lentes de Contato Especiais, Próteses Oculares e Ortoceratologia da Clínica Brunno Dantas Oftalmologia - Rio de Janeiro (RJ), Brasil;

${ }^{3}$ Livre-docente, Professor Adjunto e Chefe do Setor de Cirurgia Refrativa e Bioengenharia Ocular da Universidade Federal de São Paulo - UNIFESP - São Paulo (SP), Brasil.
}

Recebido para publicação em: 12/8/2009 - Aceito para publicação em 8/11/2009 


\section{INTRODUÇÃO}

$\Delta$ forma da superfície anterior da córnea tem relação direta com o poder final do sistema óptico humano. O poder de tal relação é grande. Sua modificação, portanto, tornou-se alvo dos procedimentos refrativos mais utilizados e populares até hoje.

Observações clínicas de pacientes usuários de lentes de contato rígidas gás permeáveis (LCRGP) chamaram a atenção para o fato de que as córneas são passíveis de deformação. Dentre as deformações, temos o aplanamento. Tais deformações, quando causadas por lentes de contato, recebem o nome de "Corneal Warpage", e hoje temos exames de imagem capazes de detectar e quantificar tal conseqüência. O "warpage" sempre foi considerado uma consequência de alterações fisiológicas e metabólicas da córnea, em decorrência de efeitos deletérios do uso de lentes de contato associados às forças mecânicas exercidas na córnea por tais lentes. Tal consideração vem sofrendo algumas modificações recentes decorrentes de estudos de biomecânica. A córnea não é tão rígida e estável como se pensava e possui propriedades viscoelásticas. Não necessariamente estas mudanças ocorrem em função de efeitos deletérios. Na fisiopatologia desta deformação inclui-se ainda o posicionamento e relação das lentes de contato com a córnea. O ápice corneano, quando deslocado, contribui para que ocorra a descentralização da lente e consequente diminuição de curvatura do mesmo. Em usuários de lente de contato rígida, a pálpebra superior pode causar um aplanamento no eixo vertical da córnea, levando a um aumento do astigmatismo. Lentes gelatinosas (LCG) muito "apertadas" ou curvas causam um aplanamento periférico, com aparente mudança refracional $^{(1)}$.

Sabemos que paciente usuário de LCRGP tem sua refração modificada após tempo prolongado do uso das mesmas e rotineiramente retira-se as $\mathrm{LC}$ ao menos 5 dias antes de avaliações pré-operatórias para candidatos a cirurgia ceratorrefrativa. Tal medida visa a determinar a forma original da córnea a ser modificada pelos excimer laser. No entanto, não há um consenso adequado e preciso de quanto tempo o paciente deve permanecer sem lentes para que se alcancem os parâmetros reais de sua córnea. Sugerimos que seja feito um acompanhamento periódico a cada 5-15 dias e verificar se está ocorrendo variação destes parâmetros. Enquanto tais variações não cessarem em três aferições seguidas no período de tempo anteriormente citado, é indicativo de que o "corneal warpage" persiste, apesar da melhora progressiva, e que se deve esperar sua total resolução. Caso ocorra a estabilização dos parâmetros, mas a córnea ainda apresente astigmatismo assimétrico e irregular e acuidade visual de óculos pior que a acuidade visual que se atingia com as lentes de contato, sobretudo as RGPs, é sugerida a investigação de ectasia corneana, um diagnóstico diferencial de "corneal warpage". Se os tratamentos forem realizados sobre uma córnea aplanada ou deformada, o resultado será menos previsível.

Geralmente, os pacientes que desenvolvem o "warpage" são usuários de lentes de contato há muitos anos e não relatam queixas, porém, alguns sinais e sintomas podem estar presentes como: hiperemia conjuntival, sensação de corpo estranho, menor tolerância ao uso de lentes de contato, visão embaçada com os óculos, marcas de compressão na córnea, deformação temporária da córnea, além de refração e ceratometria alterados ${ }^{(1)}$.

A estrutura da córnea não submetida a procedimentos cirúrgicos (pré-operatória) pode ser concebida como uma estrutura de cinco camadas submetidas à tensão, uma vez que existe uma força empurrando-os de dentro para fora (a pressão intraocular) e suas extremidades são mantidas firmemente pelo limbo.

Após a cirurgia refrativa a laser para miopia, uma série de lamelas superficiais são fotoabladas. Os segmentos restantes periféricos relaxam. Com a redução da tensão nas lamelas periféricas anteriores, a força que era exercida na matriz é reduzida e a distância entre as lamelas se expande. Isto permite o "espessamento" da periferia da córnea. Devido à forte adesão entre as camadas lamelares, a força de expansão puxa as lamelas subjacentes intactas. A força na periferia exerce tensão lateralmente sobre o centro e achata-o. Desta forma, a córnea vai achatar centralmente com qualquer procedimento circular nas lamelas. Este perfil inclui PTK, bem como o simples corte de um flap de Lasik ${ }^{(2)}$.

A ceratotomia radial (RK) ensinou-nos que a córnea é uma entidade biomecânica, e que, alterando a sua estrutura, o formato de sua superfície poderia mudar, mesmo sem a remoção de qualquer tecido. Se a córnea fosse semelhante a um pedaço de plástico homogêneo, a RK não teria função, pois uma resposta biomecânica não ocorreria após a alteração na sua estrutura. No entanto, com o desenvolvimento da cirurgia refrativa a laser, esta experiência foi considerada ultrapassada.

Nas cirurgias refrativas a laser, como o PRK ou Lasik, existem, além do efeito de aplanamento central decorrente do corte simples das lamelas, uma alteração 
da estrutura da córnea dependente do perfil de ablação, ou consumo estromal. Outro efeito biomecânico é observado quando ocorre a chamada "descompensação corneana" que pode ocorrer em casos de altas miopias tratadas com a técnica de Lasik, onde restam menos de $250 \mu \mathrm{m}$ de leito estromal residual intacto. Neste ponto, outro mecanismo assume a resposta e pode produzir ectasia corneana, ou ceratocone iatrogênico ${ }^{(2)}$.

Como objeto de diagnóstico diferencial com ectasia corneana, considera-se o efeito de lentes de contato sobre a córnea (corneal warpage). Quadros frustros de ceratocone podem se desenvolver após a retirada das lentes, e quadros observados como normais podem ser diagnosticados como ectásicos após a retirada da lente por períodos mais prolongados ${ }^{(1,3)}$.

O ceratocone é um processo degenerativo e progressivo da córnea devido à sua instabilidade biomecânica.A superfície anterior da córnea torna-se mais irregular e este processo inicia-se na idade jovem e progride gradualmente. Trata-se de uma doença geralmente bilateral e assimétrica. Sua frequência na população geral é de aproximadamente 1 em cada 2.000 pessoas $^{(3)}$. Nem sempre os óculos são eficazes na obtenção de uma boa acuidade visual, sendo assim, surgem alternativas como as LCRGP ou especiais para ceratocone, que são alternativas não invasivas, porém dependentes de uma adaptação por parte do paciente. Quando não se consegue o efeito esperado, alternativas cirúrgicas podem ser utilizadas. É o caso dos transplantes de córnea penetrantes e lamelares e os anéis intraestromais. Muitas vezes, após tais procedimentos cirúrgicos, faz-se necessário a adaptação de lentes de contato especiais para se obter a melhor acuidade visual possível.

Tratamentos como transplantes lamelares e implante de anéis intraestromais são possibilidades cirúrgicas, porém estas técnicas supõem que o estroma corneano se manterá estável após a aplicação. Sabe-se que em muitos casos, este encontra-se vulnerável ${ }^{(3)}$.

Visando a preservar e fortalecer o estroma da instabilidade biomecânica, foi desenvolvida uma técnica que envolve a promoção das ligações covalentes de colágeno nesta região. Esta técnica é chamada de "Crosslink" de colágeno corneano - Ultra B2 ou simplesmente CXL. Utiliza-se vitamina B2 (Riboflavina) que atua como elemento foto-sensível, e juntamente com a luz ultravioleta promove novas ligações covalentes intra e interfibrilares, através da oxidação foto-sensível, tornando a córnea mais rígida e menos elástica ${ }^{(3)}$. No caso do ceratocone, este enrijecimento da córnea poderá retardar seu avanço, porém não consegue revertê-lo.
A utilização de ablações personalizadas em casos de ectasia e deformação corneana é um campo em desenvolvimento. Até então, sabemos que a retirada de mais tecido (ablação) de córneas com estrutura alterada (ceratocone) carrega maior risco de descompensação e aceleração da sua deformação. A promoção de novas ligações covalentes entre as lamelas da córnea (CXL) tem sido apontada como fato novo a modificar o conceito atual.

\section{A Ortoceratologia}

O processo de manipular o olho para mudar ou eliminar erros refrativos teve sua origem com um jovem chinês que pressionava sacos de areia sobre os olhos para mudar seu estado refrativo ${ }^{(4,5)}$. Após estudos sobre a miopia realizados em 1956 por Robert Morrison percebeu-se que os pacientes adolescentes que usavam lentes de PMMA com curvatura posterior mais plana que a curvatura anterior de sua córnea tiveram a miopia "estabilizada". Usava-se lentes rígidas de PMMA para aplanar a córnea e conseguir um resultado desejado de correção do erro refrativo. Várias tentativas ocorriam até que se alcançasse o objetivo, acontecendo algumas grandes imprevisibilidades, pelo mau posicionamento da lente ${ }^{(6)}$.

Um estudo, desenvolvido por Grant e May, baseou-se em adaptar lentes com a curva base igual ou $0.37 \mathrm{~mm}$ mais plana que a ceratometria mais plana da córnea. O resultado foi um aplanamento corneano que eliminava ou diminuía a ametropia dos pacientes. Segundo seus relatos: "O tempo de uso da lente era gradativamente diminuído até que o uso das lentes era eliminado por completo" (4).

A lente de ortoceratologia altera o formato anterior da córnea mais que o raio de curvatura posterior. Estes achados sugerem que o fator primário no efeito refrativo é a mudança no formato da córnea anterior mais que toda a curva corneana ${ }^{(7)}$.

A utilizacão clínica da deformação corneana induzida pelas lentes de contato forma a base da ortoceratologia( $^{(8)}$.

A ortoceratologia foi descrita pela primeira vez em 1962, no segundo congresso mundial de lentes de contato $^{(9)}$. A Divisão Internacional de ortoceratologia (IOS) da Fundação Nacional de Pesquisa em Oftalmologia (NERF) definiu a ortoceratologia como a redução ou mesmo eliminação da miopia, hipermetropia e/ou do astigmatismo pela adaptação programada de lentes de contato de desenho específico para cada paciente $(4,6,8,9,10,11,12)$.

Já no final dos anos 70, surgiram os materiais 


\section{Quadro 1}

\section{Comparação entre a ortoceratologia clássica e "avançada" (6)}

\begin{tabular}{ll}
\hline Antiga & Nova \\
\hline Material das lentes feito de PMMA & $\begin{array}{l}\text { Lentes de geometria inversa e material gás permeável de alta permeabilidade } \\
\text { (ISSO/Fatt Dk de 85 ou mais) que permite o uso durante o sono. }\end{array}$ \\
$\begin{array}{l}\text { Resultados demorados e imprecisos } \\
\text { Baixo controle e pouca precisão } \\
\begin{array}{l}\text { sobre o posicionamento da lente } \\
8 \text { ou mais pares de lentes na tentativa de } \\
\text { definir a melhor lente }\end{array}\end{array}$ & $\begin{array}{l}\text { Melhor controle e precisão sobre o posicionamento da lente } \\
\text { Bou segundo par de lentes }\end{array}$ \\
\hline
\end{tabular}

rígidos gás permeáveis e foram confeccionadas lentes maiores com a finalidade de obter uma melhor centralização para alcançar melhores resultados. Porém, a imprevisibilidade ainda prevalecia, bem como a preocupação com a alteração corneana e com o tempo de tratamento. Nesta mesma época, estudos comprovavam que o risco de complicações com as lentes convencionais eram os mesmos que com as lentes de ortoceratologia $^{(10,13)}$.

Nos anos 80 e 90 surgiram os tornos computadorizados e as lentes de geometria reversa. Acredita-se que Richard Wlodyga finalmente tenha desenvolvido as lentes de ortoceratologia. Em 1993 tratamentos desta lente com uso diurno foram relatados ${ }^{(10)}$.

\section{Ortoceratologia atual: definições e conceitos}

Em outras palavras, a ortoceratologia (ortoK) é um procedimento não cirúrgico que permite o remodelamento ou aplanamento corneano através de lentes de contato, a fim de reduzir os erros refrativos $(4,6,8,9,11,12,14,15)$.

Apesar das cirurgias refrativas serem muito difundidas, a ortoK acaba sendo escolhida por diversos pacientes, por ser considerada uma técnica segura e reversível $^{(4)}$. Com o advento de novos modelos e inovações nas lentes de contato, o interesse em ortoK cresceu consideravelmente ${ }^{(4,11)}$.

Os avanços na obtenção de maior transmissibilidade de oxigênio DK/L influenciaram na prática da ortoK. A menor transmissibilidade ao oxigênio que a lente precisa ter para que o paciente tenha certa segurança durante o sono, é de $87 \mathrm{Dk} / \mathrm{L}$ ( $\mathrm{cm}$ x mL x O2) / ( $\operatorname{seg}$ x mL x mmHG). Novas gerações de materiais possuem o Dk/L suficiente para não causar hipóxia corneana e permitir seu uso durante o sono ${ }^{(16)}$.

$\mathrm{Na}$ ortoceratologia moderna, o remodelamento corneano ocorre com maior rapidez, proporcionando uma alteração significativa já na primeira noite de uso, tendo seu melhor resultado após 30 dias de tratamento. Usam-se, agora, lentes de geometria reversa. A quantidade a ser aplanada na córnea é controlada, permitindo uma maior chance de sucesso durante a adaptação ${ }^{(6)}$. As lentes de contato rígidas com curva de geometria reversa possuem um aplanamento central com um "degrau" que envolve outra curva. A curva central exerce pressão positiva na córnea, induzindo ao aplanamento ${ }^{(8)}$.

Com o tempo, várias empresas desenvolveram as lentes de 3, 4 e 5 curvas com geometria reversa e com desenhos de periferia esférica, asférica e tangencial e um novo termo surgiu para definir a nova era em terapia: "Ortoceratologia Avançada" (10,14).

No quadro 1 apresentamos uma comparação entre a ortoceratologia clássica e "avançada"

Existem muitas teorias sobre o mecanismo de ação da lente de ortoceratologia ${ }^{(10)}$. Sabe-se que existe uma pressão do filme lacrimal na córnea e ocorre um deslocamento das células epiteliais durante o sono principalmente $^{(10,12)}$. O filme lacrimal fica entre a lente de contato e a córnea, e esta camada pode exercer uma força positiva ou negativa sobre a córnea, dependendo de como ela está distribuída ao longo da superfície. No local onde a camada lacrimal fica mais espessa, esta força é menor que no local onde a camada é mais fina e a lente se aproxima mais do epitélio. Com a proximidade da face posterior da lente e anterior da córnea ocorre a pressão positiva, que irá deslocar as células epiteliais, redistribuindo-as até ocorrer um equilíbrio na superfície corneana ${ }^{(10)}$.

O epitélio corneano possui, em média, 50um de espessura e 5-6 camadas de células, que serão comprimidas e se deslocarão. O deslocamento reversível do epitélio é o responsável pela alteração refrativa ${ }^{(10)}$. A camada epitelial central torna-se mais delgada, enquanto que a camada da média periferia torna-se mais espessa. ${ }^{(11,12)}$. As células do epitélio, que são em grande parte compostas por água, se comportarão como um fluido 


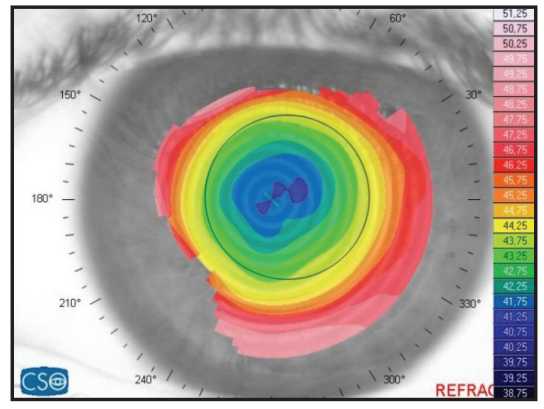

Figura 1: Imagem topográfica em alvo ${ }^{(18)}$

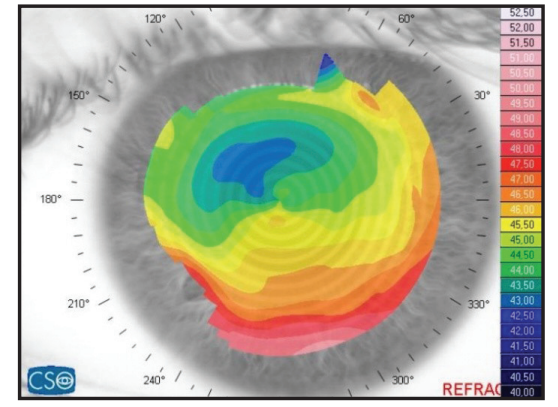

Figura 2: Face sorridente ${ }^{(18)}$

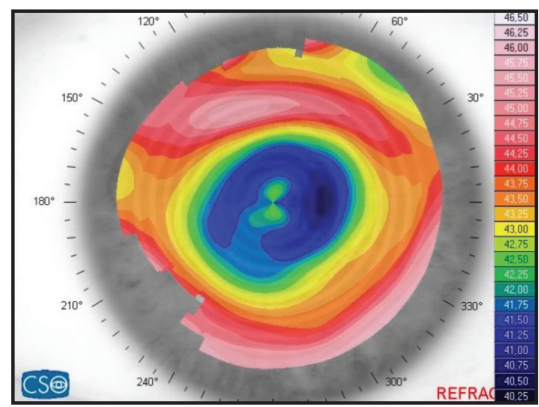

Figura 3: Ilha central ${ }^{(18)}$
Newtoniano, obedecendo ao princípio de Pascal: quando uma força é aplicada igualmente sobre uma superfície, estando dentro de um sistema fluido fechado, a pressão é uniforme. Porém, quando esta força é aplicada de forma desigual, como no caso do filme lacrimal e o epitélio, então o tecido será deslocado até que se tenha um equilíbrio tecidual. Isso é possível acontecer por ser a superfície da córnea um sistema aberto ${ }^{(10)}$. O volume total do epitélio não se altera, porém ocorre um deslocamento da área central até a área paracentral. Este afinamento central é de no máximo $20 \mu \mathrm{m}$, ocasionando a diminuição do poder refrativo, com resultados variáveis dependendo do diâmetro da área tratada ${ }^{(10)}$.

O conceito de excentricidade (Valor-E) é importante no entendimento dos princípios da ortoK. Essa seria a diferença entre a curvatura apical da córnea e da sua periferia. É o quanto a córnea ainda poderia ser aplanada do ápice até a periferia. Quanto maior este valor (até determinado ponto), maior vai ser a alteração que a córnea pode sofrer. $\mathrm{O}$ paciente ideal deve ter um valor positivo de excentricidade $(0,40-0,45)$. Córnea esférica, ou seja, sem excentricidade, tem o Valor-E igual a 0,0 .

Não é aconselhado, portanto, indicar a terapia da ortoKpara pacientes que possuem altas prescrições e baixos valores de excentricidade (Valor $-\mathrm{E})^{(10)}$.

Outros dados topográficos necessários para a adaptação da orto incluem: 1) raio apical, entendido como o raio de curvatura no ápice da córnea. Este valor deve ser muito preciso já que um erro de $0.01 \mathrm{~mm}$ no raio apical (Ro) é igual a $2 \mu \mathrm{m}$ em um erro na altura sagital ${ }^{(10)}$; e 2) Altura sagital, que é a altura da córnea (medida em mícron) sobre um diâmetro de corda. O comprimento de corda não pode ser menor que $9.35 \mathrm{~mm}^{(10)}$.

A adaptação da lente de ortoK é um procedimento que envolve a interpretação dos mapas topográficos corneanos ${ }^{(10,17)}$, análise do padrão de fluoresceína (dependendo do programa utilizado), além do frequente acompanhamento $^{(17)}$. Deve-se selecionar o paciente sabendo-se o seu Valor-E, Raio apical (RO) e refração ${ }^{(10)}$. A topografia corneana indica alterações corneanas du- rante a ortoK, e 3 padrões podem ser observados a saber:

1) Imagem topográfica em alvo: é o resultado esperado da topografia corneana para a resposta refrativa padrão em um caso bem-sucedido de ortoK. A zona de tratamento deverá ser compatível com o tamanho pupilar sob iluminação fraca. No mapa refrativo, esta zona aparecerá, geralmente, como uma área esverdeada ou azulada, profunda e centralizada sobre "o ápice". Ou seja, será observado um aplanamento central (figura 1). No período de adaptação, o paciente poderá apresentar uma pequena sobrerrefração que poderá ser corrigida temporariamente com a adaptação de uma lente hidrofílica. Esta sobrerrefração é inserida no programa da lente e ao programar a confecção da lente, esta terá seu parâmetro corrigido ${ }^{(10)}$.

2) Face sorridente: resultado corneano negativo devido a uma adaptação plana de lentes de ortoK. No mapa refrativo aparece uma imagem vermelho/ alaranjado na região inferior à pupila (figura 2). Para confirmar os achados, o mapa tangencial confirma o posicionamento superior ou incompleto da lente na região pupilar, ocasionando uma acuidade visual indesejada. O teste poderá ser refeito após 2-3 dias da readaptação de nova lente de ortoK ${ }^{(10)}$.

3) Ilha central: ocorre nos casos em que a lente está apertada, causando redistribuição inversa do epitélio, que irá aplanar a periferia e encurvar o centro da córnea (figura 3). É uma resposta inadequada ao tratamento. O mapa topográfico refrativo tem a aparência de uma ilha central no ápice corneano ${ }^{(10)}$.

Outras imagens podem também serem vistas, geralmente mistas, e decorrentes, na maioria das vezes, do deslocamento da lente no olho do paciente, durante o sono.

A complexidade da adaptação das lentes depende do reconhecimento dos padrões de deformação, que tem relação com a experiência do observador. Tal processo restringiu a disseminação da técnica, que possui bases promissoras, a poucos centros no mundo. As empresas produtoras de lentes recentemente propuseram estratégias novas para facilitar a adaptação. 
Pensou-se recentemente que, com a evolução de softwares modernos, a análise topográfica e os testes com fluoresceína tornar-se-iam dispensáveis, já que os programas instalados nos topógrafos permitem a medição dos dados corneanos e a escolha da lente indicada para o paciente. No entanto, vimos que isto não ocorreu. Tais softwares ainda não são tão precisos e estão longe de substituir o profissional humano. Eles partem do princípio que o olho e seus anexos são estáticos, e não levam em consideração a biomecânica corneana. Baseiam-se em dados retirados do resultado de exames, passíveis de erros (mesmo que pequenos) e não raciocinam por si só. Não levam em consideração a posição de dormir do paciente, bem como sua qualidade de sono. Alguns pacientes não respondem bem à ortoceratologia em razão de pequenos deslocamentos da lente no olho causado por determinadas posições durante o sono. Os softwares não estão aptos para compensar tais contratempos. Pelo menos, não ainda.

\section{Abstract}

The cornea has a great influence in the visual process. However, it is liable to distortion, such as flattening. When this flattening is caused by the use of contact lenses, we call "corneal warpage". In this case, some symptoms may occur as blurred vision with glasses, conjunctival hyperemia, foreign body sensation, etc... Used to think that the cornea was more rigid and, in time, realized that the cornea has viscoelastic properties. We can say that the cornea has a body biomechanics property and suffers instability when there are some changes, such as keratoconus, which is a progressive corneal degeneration. In order to preserve the cornea more stable, the cross-linking technique was developed to promote covalent bonds between the lamellae of the cornea to make it stronger and less elastic. Other corneal change that may occur is based on the induction in corneal reshaping by using rigid contact lenses (only during sleeping) with high oxygen transmissibility $(D K / L)$. This technique is known as Orthokeratology and its concepts will be addressed in this study.

Keywords: Orthokeratologic procedures; Orthokeratology; Collagen; Corneal topography; Corneal physiopathology; Cornea/injuries; keratoconus; Contact lenses; Riboflavin/therapeutic use; Ultraviolet therapy

\section{REFERÊNCIAS}

1. Lipener C. Deformidade corneana em usuário de lente de contato portador de ceratocone: relato de caso. Arq Bras Oftalmol. 1999; 62(6):713-6.

2. Roberts, C. The cornea is not a piece of plastic. J Refract Surg. 2001;17(1):76-7.

3. Jankov MR $2^{\text {nd }}$, Hafezi F, Beko M, Ignjatovic Z, Markovic V, Schor P. Corneal Cross-linking for the treatment of keratoconus:preliminary results. Arq Bras Oftalmol. 2008; 71(6):813-8.

4. Joe JJ, Marsden HJ, Edrington TB. The relationship between corneal eccentricity and improvement in visual acuity with orthokeratology. J Am Optom Assoc. 1996;67 (2): 87-97.

5. Pearson RM. Kalt, keratoconus and the contact lens. Optom Vis Sci 1989;66(9): 643- 6.

6. Ortoceratologia. Available from: http:www.eyetec.com.br. Acessed dez 2008.

7. Tsukiyama J, Miyamoto Y, Higaki S, Fukuda M, Shimomura Y. Changes in the anterior and posterior radii of the corneal curvature and anterior chamber depth by orthokeratology. Eye Contact Lens. 2008; 34(1):17-20.

8. Young AL, Leung AT, Cheng LL, Law RW, Wong AK, Lam DS. Orthokeratology lens- related corneal ulcers in children: a case series. Ophthalmology. 2004; 111(3): 590-5.

9. Grant SC. Orthokeratology. I. A safe and effective treatment for a disabling problem. Surv Ophthalmolol. 1980;24(5):291-7.

10. Mountford J, Noack D. Design be free [Internet]. São Paulo: Mediphacos. [cited 2007 Dec 15]. Available from: http:// www.ortobefree.com.br.

11. Wang J, Fonn D, Simpson TL, Sorbara L, Kort R, Jones L. Topographical thickness of the epithelium and total cornea after overnight wear of reverse- geometry rigid contact lenses for myopia reduction. Invest Ophthalmol Vis Sci. 2003;44(11):4742- 6 .

12. Alharbi A, Swarbrick HA. The effects of overnight orthokeratology lens wear on corneal thickness. Invest Ophthalmol Vis Sci. 2003; 44(6): 2518- 23.

13. Stillitano I. Ortoceratologia [tese]. São Paulo: Universidade Federal de São Paulo; 2007.

14. Ramkisson P. A clinical evaluation of overnight orthokeratology as a method of vision correction. S Afr Optom. 2004;63:167-168.

15. Chen KH, Kuang TM, Hsu WM. Serratia Marcescens Corneal Ulcer as a complication of orthokeratology. Am J Ophthalmol. 2001; 132(2): 257-8.

16. Liang JB, Chou PI, Wu R, Lee YM. Corneal iron ring associated with orthokeratology. J Cataract Refract Surg. 2003; 29(3): 624-6.

17. Garber JM. Overnight orthokeratology. Optom Vis Sci. 2001;78(7):480.

18. Godinho CJ, Dantas B, Sobrinho M, Polisuk P. O padrão CG em lentes de contato. Rio de Janeiro: Cultura Médica, 2008. p.208-10. 